Bol. Soc. Bot. México 50:121-133(1990)

\title{
Caracterización ficoflorística de los paredones de la Sierra de Juárez, Oaxaca. Importancia de las formas de crecimiento algales en la tipificación de un ambiente.
}

\author{
Rosa Luz TAVERA-SiERrA y JORGE GONZÁleZ-GONZÁLEZ ${ }^{1}$
}

\begin{abstract}
RESUMEN. En este estudio se muestra la importancia de integrar la fisiografía de un ambiente con la manifestación conspicua de las algas, a la que llamamos forma de crecimiento, para caracterizar dicho ambiente utilizando las formas de crecimiento que lo tipifican. Con este criterio se analizaron los paredones de la Sierra de Juárez encontrándose las siguientes formas de crecimiento: crecimientos hemisféricos compactos, flóculos filamentosos, flóculos mucilaginosos, películas compactas, películas filamentosas, tapetes y costras. Estas formas se describen en 15 muestras. 53.3\% son de tipo floculoso y de ellas el 33.3\% son flóculos filamentosos y el $20 \%$ son flóculos mucilaginosos. Las descripciones de la cobertura algal y de la fisiografía de paredones que hemos hecho para la Sierra de Juárez, son muy similares a las de otras latitudes, en las que predominan también los crecimientos floculosos, lo que nos permite definir un paredón como escurrimientos verticales con muy poca cantidad de agua en los que las formas de crecimiento típicas son los flóculos filamentosos y los flóculos mucilaginosos.
\end{abstract}

ABSTRACT. This study shows the importance of integrating the physiography of a community with the visible manifestation of algae, that we call growth form, to characterize communities in terms of the growth forms that typify it. The drip walls of Sierra de Juárez were analyzed with this approach. The growth forms present are: compact hemispherical growths, filamentous flocculus, mucilaginous flocculus, compact films, filamentous films, nugs and crusts. These forms are described from 15 samples. $53.3 \%$ of the flocculus type, $33.3 \%$ of these are filamentous and $20 \%$ are mucilaginosus. Our descriptions of the algal cover and physiography of the Sicrra de Juárez are very similar to the descriptions of drip walls in other

${ }^{1}$ Laboratorio de Ficología, Facultad de Ciencias, Universidad Nacional Autónoma de México. Apdo. Postal 70-620, Delegación Coyoacán; 04510. México, D.F.

Tavera-Sierra RL, González-González J. 1990. Caracterización ficoflorística de los paredones de la Sierra de Juárez, Oaxaca. Importancia de las formas de crecimiento algales en la tipificación de un ambiente. Boletín de la Sociedad Botánica de México 50: 121-133. 
latitudes, also with a predominance of flocculus growths. We define a drip wall as a vertical wall with not very much water and filamentous and mucilaginous flocculus as typical growth forms.

Los estudios florísticos sobre algas se conciben como un quehacer particular de los taxónomos, y por ende los procedimientos que se utilizan y los conceptos con los que se trabaja se encuentran incluidos en los marcos de la taxonomía y frecuentemente separados de los marcos y procedimientos de otras áreas como la ecología y la biogeografía. Así, si el objeto de estudio es la flora algal, como un colectivo, no se debe trabajar sólo con procedimientos taxonómicos porque las algas son un grupo funcional. Para elaborar la caracterización ficoflorística de una región se debe integrar la ficoflora presente con las características fisiográficas y ambientales.

Para hacer esta integración consideramos la manifestación conspicua de las algas, es decir cómo crecen visiblemente, como una característica fisiográfica del ambiente a la que llamamos forma de crecimiento.

\section{MEtodologíA}

Las muestras fueron colectadas en la Sierra de Juárez, en la parte norte del estado de Oaxaca. La ubicación se muestra en la figura 1, el mapa de localidades. Las muestras se encuentran depositadas en el Herbario de la Facultad de Ciencias, UNAM (FCME), con el nombre de la colección del proyecto Flora fícológica de la cuenca del río Papaloapan (PAP).

En el sitio de colecta cada crecimiento visible fue ciescrito morfológicamente, anotando el $\mathrm{pH}$, temperatura del agua, el tipo de sustrato y el grado de humectación de cada punto de colecta. También se anotaron las características generales del ambiente tales como orientación, tipo de sustrato geológico de la localidad y vegetación acompañante (cuadro 1). Esta última se detalla sólo como un elemento adicional de información sobre el ambiente. La referencia a esta vegetación perfila un cierto grado de heterogeneidad de las localidades ya que se trata sólo de las plantas que crecen sobre los paredones, aunque tal referencia pueda resultar inadecuada para las formas de vida y/o aspectos taxonómicos de las plantas en cuestión.

Cada muestra fue revisada al microscopio con varias preparaciones. Para cada preparación se tomó una porción de la forma de crecimiento contenida en la muestra, para determinar todas las especies presentes. E⿺ número de preparaciones varió para cada muestra; una muestra se consideró revisada cuando ya no se encontraron especies diferentes.

Para cada forma de crecimiento se estimó la abundancia relativa de las especies constituyentes, asignando siempre el númcro uno (1) a la especie más abundante, por ello las especies aparecen numeradas en un orden progresivo. En el cuadro 2 se señala la especie con mayor abundancia relativa para cada forma de crecimiento, la muestra 
CUADRO 1. Características fisiográficas y formas de crecimiento de los paredones de la Sierra de Juárez, Oaxaca, en cada localidad.

\begin{tabular}{|c|c|c|c|c|c|c|c|c|}
\hline LOCALIDAD & $\begin{array}{l}\text { CARACTERISTICAS } \\
\text { DE HUMEDAD }\end{array}$ & $\begin{array}{c}\text { TIPO DE } \\
\text { SUSTRATO* }\end{array}$ & $\begin{array}{l}\text { VEGETACION } \\
\text { ACOMPAÑANTE }\end{array}$ & $\begin{array}{l}\text { FORMA DE CRECIMIENTO } \\
\text { ALGAL }\end{array}$ & $\mathrm{pH}$ & $\begin{array}{l}\text { TEMPERATURA } \\
\text { DEL AGUA }\left({ }^{\circ} \mathrm{C}\right)\end{array}$ & ORIENTACION & $\begin{array}{r}\text { ALTITUD } \\
\text { (msnm) }\end{array}$ \\
\hline Teotitlán & $\begin{array}{l}\text { escurrimiento } \\
\text { escaso }\end{array}$ & RSM tipo "A" & $\begin{array}{l}\text { helechos, mus- } \\
\text { gos, hierbas. }\end{array}$ & $\begin{array}{l}\text { tapetes, crecimientos } \\
\text { hemisféricos compactos, } \\
\text { flóculos filamentosos, } \\
\text { flóculos mucilaginosos, } \\
\text { películas filamentosas. }\end{array}$ & 6.5 & 21 & $90^{\circ} \mathrm{E}$ & 1450 \\
\hline Huautla & goteo & $\begin{array}{l}\text { RSM tipo "B" } \\
\text { gos, hepáticas }\end{array}$ & helechos, mus- & $\begin{array}{l}\text { costras, } \\
\text { flóculos filamentosos. }\end{array}$ & 6.0 & 15 & $260^{\circ} \mathrm{SW}$ & 2000 \\
\hline $\mathrm{Km} 24$ & $\begin{array}{l}\text { escurrimiento } \\
\text { de capa delgada }\end{array}$ & RSM tipo "B" & $\begin{array}{l}\text { hierbas, mus- } \\
\text { gos }\end{array}$ & $\begin{array}{l}\text { flóculos filamentosos, } \\
\text { películas compactas. }\end{array}$ & 6.5 & 19 & $27.4^{\circ} \mathrm{NE}$ & 1725 \\
\hline $\begin{array}{l}\text { San Juan } \\
\text { Coatzospan }\end{array}$ & filtración & $\begin{array}{l}\text { Roca sedimen- } \\
\text { taria tipo "C" }\end{array}$ & $\begin{array}{l}\text { musgos, hepá- } \\
\text { ticas, líquenes }\end{array}$ & $\begin{array}{l}\text { flóculos mucilaginosos, } \\
\text { flóculos filamentosos }\end{array}$ & 6.0 & 18 & $18^{\circ} \mathrm{NE}$ & 1727 \\
\hline
\end{tabular}

* RSM: roca sedimentaria metamorfizada. Tipo "A": rocas de color gris claro a pardo oscuro, compuestas de filitas y arcosas, que descansan sobre gneisses. Tipo "B": rocas blanquecinas compuestas de calizas dolomíticas, calcarenitas, lutitas y areniscas. Tipo "C": calizas puras y yesífcras, calizas dolomíticas y calcarenitas. 
CUADRO 2. Especies que determinan cada forma de crecimiento

FORMA DE CRECIMIENTO

TEOTITLAN

Gomphosphaeria aponina (323)

Gloeothece rupestris (328-1)

compactos

Flóculos filamentosos

Flóculos mucilaginosos

Películas compactas

Películas filamentosas

Tapetes

Costras
HUAUTLA

$\mathrm{Km} 24$

SAN JUAN COATZOSPAN
Oedogonium sp.1(345)

Mougeotia drouetii (246-1)

Zygnema sp. (348)

Aphanothece naegelli (324-3)

Anabaena sp. (328-2a)

Microcystis marginqta (328-2b)

Microcystis protocystis (324-4)

Chrysosaccus epilithicus (325)

Rhizoclonium hieroglyphicum (326-1)

Oscillatoria lemmermannii (326-2)
Mougeotia drouetii (347)
Mougeotia drouetii (349-2)

Aphanocapsa muscicola (349-1)

Ordenadas por localidad. Solamente se menciona la especie con mayor valor de abundancia relativa en cada forma de crecimiento. Las especies están acompañadas por un paréntesis que indica el número de muestra en que se encuentran. 
en la que se encuentra y la localidad a la que corresponde. En los resultados, junto al nombre cada especie se indica su valor de abundancia. En cada muestra, los valores de abundancia representados por estos números fueron asignados de manera independiente de las demás muestras, es decir, no son comparables las abundancias relativas de las especies entre una y otra muestra. Las formas de crecimiento no son equivalentes en ese sentido. La asignación de estos valores se hace para resaltar que las características que definen una forma de crecimiento no están dadas por la presencia de determinadas especies sino por algunas cualidades en la manifestación de las mismas, por ejemplo la abundancia. La terminología para las formas de crecimiento es la utilizada en la Universidad de Durham (Díaz et al., 1976; Donaldson y Whitton, 1977; Donaldson, 1978; Whitton et al., 1979).

\section{DESCRIPCIÓN GENERAL DEL ÁREA DE ESTUDIO}

El área de estudio es parte de la franja montañosa de la Sierra Madre Oriental y las rocas son principalmente sedimentarias del Mesozoico (Salas, 1977). La zona que comprende este trabajo se localiza entre los $18^{\circ} 00^{\prime}$ y $18^{\circ} 15^{\prime}$ de latitud norte, y entre los $96^{\circ} 20^{\prime}$ y $97^{\circ} 10^{\prime}$ de longitud oeste. El clima es Am (cálido-húmedo con lluvias en verano); la temperatura media y precipitación anuales varían desde $24^{\circ} \mathrm{C}$ y $298 \mathrm{~mm}$ respectivamente (a $564 \mathrm{msnm}$, en Cuicatlán); hasta $15.6^{\circ} \mathrm{C}$ y $1000 \mathrm{~mm}$ respectivamente (a 2000 msnm, en Huautla) (Mosiño, 1977). La vegetación también varía con la altitud. La Sierra de Juárez se caracteriza por un bosque mixto de pino-encino, en el que Pinus montezumae o Pinus oocarpa y Quercus conspersa son las especies más frecuentes y en el sotobosque son muy abundantes las ericáceas como Gaultheria hirtiflora y Vaccinium geminiflorum (Miranda, 1948). En las partes más altas y húmedas del área de colecta es notoria la abundancia de briofitas y pteridofitas. (fig. 1).

\section{RESUlTADOS}

De las 16 muestras que constituyen la colecta analizada en este trabajo, 15 corresponden a paredones. En ellas el $53.3 \%$ de las formas de crecimiento son de tipo floculoso, y de éstas el $33.3 \%$ son flóculos filamentosos y el $20 \%$ son flóculos mucilaginosos (cuadros 1 y 2 ).

TEOTITLAN Ambiente mixto formado por un arroyo y paredones. Esta localidad es la ladera de la montaña que se corta en la carretera a 6 km hacia el NE de Teotitlán del Camino, Oaxaca (18 ${ }^{\circ} 10^{\prime} 17.24^{\prime \prime}$ lat N; 97 $3^{\prime} 15.36^{\prime \prime}$ lon O). Presenta un sustrato rocoso sedimentario metamorfizado, (rocas de color gris claro a pardo oscuro, compuestas de filitas y arcosas que descansan sobre gneisses). Tanto las partes verticales (paredones) como las horizontales (arroyo) tienen depósitos de lodo que constituyen también un sustrato para los crecimientos algales. El afloramiento de agua es escaso y escurre de manera continua. Sobre la pared crece una vegetación relativamente abundante formada 


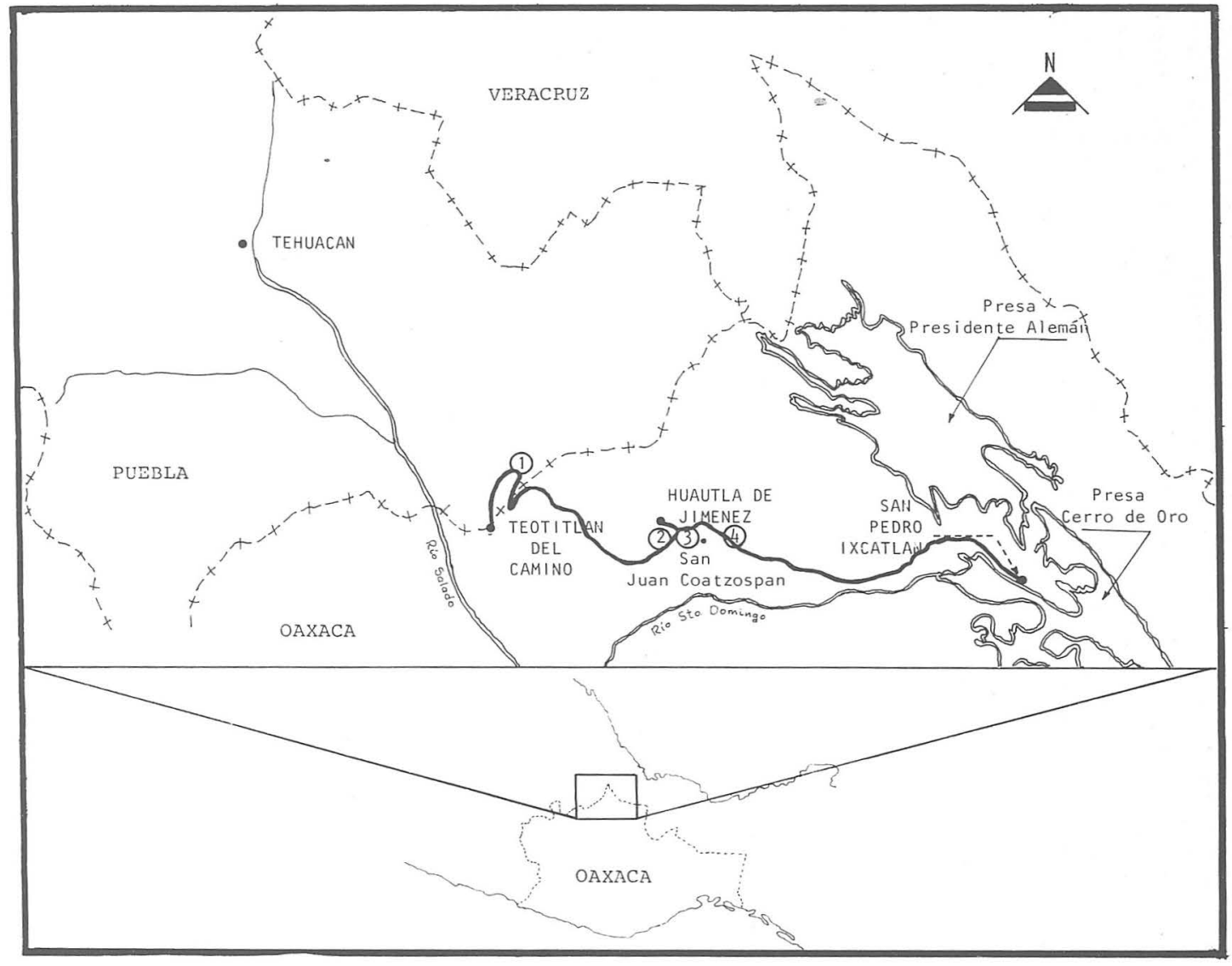

Fig. 1 Mapa de localidades. Ubicación de las localidades muestreadas en la Sierra de Juárez, Oaxaca. 1. Teotitlán; 2. Huautla 3.km 24; 4. San Juan Coatzospan. 
por algunas hierbas, helechos y musgos. El panorama ficológico es diverso y exuberante compuesto de flóculos, películas, y crecimientos hemisféricos compactos principalmente. El pH es de 6.5 y la temperatura del agua es de $21^{\circ} \mathrm{C}$. La orientación es de $90^{\circ} \mathrm{E}$ y se encuentra a $1450 \mathrm{msnm}$.

PAP 323 (FCME) Forma de crecimiento: crecimiento hemisférico compacto.

El arroyo tiene sustrato rocoso con suelo depositado y algunos guijarros. La profundidad es variable, con un máximo de $10 \mathrm{~cm}$. Cubriendo los guijarros del fondo se encuentran unos crecimientos hemisféricos compactos de color verde claro y textura suave. Estos crecimicntos son masas compactas de mucilago en forma de domos translúcidos, lo que les da apariencia lustrosa; son muy pequeños (1 ó $2 \mathrm{~mm}$ ). Cuando crecen en un paredón, es más común encontrarlos como crecimientos subaéreos, fuera de los escurrimientos, véase PAP 328-1 (FCME). Las especies que integran la forma de crecimiento de esta muestra son: 1 Gomphosphaeria aponina; 2 Oscillatoria foreaui; 3 Protoderna vivide; 4 Lyngbya kuetzingii; 5 Oscillatoria tenuis; 6 Phormidium ambiguum; 7 Calothrix elenkinii; 8 Chaetonema irregulare; 9 Navicula lanceolata; 10 Epithemia argus; 11 Nitzschia hantzschiana; 12 Synedra ulna. Las siguientes muestras fueron colectadas en las paredes; en éstas, el sustrato sobre el que crecen las algas es rocoso, pero la roca puede estar cubierta en partes por lodo y musgos, así que hay crecimientos epilíticos, epipélicos y epífitos cuyas diferencias florísticas se anexan a la descripción de las siguientes muestras.

PAP 324-1 y 324-2 (FCME) Forma de crecimiento: flóculo filamentoso.

En el escurrimiento de agua se encuentran flóculos filamentosos de color verde oscuro y textura rasposa. Crecen adheridos a la roca, sobre lodo depositado y musgos, los cuales forman manchones dispersos, pero siempre en sitios de escurrimiento. Hemos designado como flóculo filanentoso a la forma compuesta de filamentos que crecen entremezclados, de una consistencia lanosa y que alcanzan un volumen notable. Esta forma de crecimiento es común en los paredones y crece sobre distinto tipo de sustratos. Véase PAP 345 (FCME), PAP 346-1 (FCME), PAP 348 (FCME). En esta forma de crecimiento se encuentran las siguientes especies: 1 Vaucheria sp.; 2 Amphora ovalis; 3 Denticula elegans; 4 Gloeothece nupestris; 5 Gloeothece sp.; 6 Achnanthes hauckiana; 7 Achnanthes lanceolata 8 Achnanthes grimmei; 9 Achnanthes exigua; 10 Cosmarium margaritatum; 11 Cosmarium formosulum 12 C. formosulum var. hyperevolutum; 13 Gloeocapsa polydematica; 14 Cosmarium pseudopyramidatum; 15 Chroococcus minor; 16 Microcystys aenuginosus; 17 Cosmarium pachydernum var. complanatum; 18 C. pachydermum var. pusillum; 19 Oocystis crassa; 20 Pediastrum integrum; 21 Nitzschia hantzschiana; 22 Mastogloia elliptica var. dansei; 23 Chroococcus turgidus; 24 Navicula lanceolata; 25 Navicula salinarum var. intermedia; 26 Navicula cincta; 27 Navicula pupula. var. capitata; 28 Lyngbya perelegans; 29 Synedra ulna; 30 Oscillatoria sancta.

PAP 324-3 (FCME) Forma de crecimiento: flóculo mucilaginoso.

Fuera de la corriente del escurrimiento, humedecido por el rocío, se encuentra un flóculo mucilaginoso de color verde claro y textura suave, creciendo sobre la roca. Un 
flóculo murcilaginoso tiene consistencia muy acuosa y crece muy laxamente. Es una forma de crecimiento subaérea por excelencia. En los paredones puede alcanzar algunos centímetros de espesor y puede llegar a ser un crecimiento exuberante (véase PAP 349-1 (FCME). Se adhiere al sustrato por el mucilago mismo y se desprende muy facilmente. Esta forma de crecimiento está constituida por: 1 Aphanothec naegelii; 2 Microcysti protocystis; 3 Aphanothece castagnei; 4 Lyngbya perelegans; 5 Gloeothece rupestris; 6 Denticula elegans; 7 Oocystys crassa; 8 Aphanothece caldariorum; 9 Cosmarium formosulum; 10 Cosmarium pseudopyramidatum; 11 Oedogonium sp. 1; 12 Pediastrum integrum; 13 Amphora ovalis; 14 Achnanthes grimmei.

PAP 324-4 (FCME) Forma de crecimiento: película compacta.

Fuera de la corriente del escurrimiento y humedecida por el rocío, se encuentra creciendo sobre la roca una película compacta de color verde claro y textura mucilaginosa. En la película compacta no se distingue una trama filamentosa (aunque el nivel de organización de la o las especies que la conforman sea.filamentoso), a menos que se observe a más de 25 aumentos. Tampoco tiene la consistencia lanosa del flóculo filamentoso, o el espesor, de la película filamentosa. Se manifiesta como una piel que recubre las rocas; siempre se desprende sin rasgarse. Esta forma de crecimiento está constituida por: 1 Microcystis protocystis; 2 Oocystis crassa; 3 Microcystis pulverea; 4 Lyngbya perelegans; 5 Gloeothece rupestris; 6 Chroococcus sp.

PAP 325 (FCME) Forma de crecimiento: película compacta.

El escurrimiento forma una pequeña caída de agua y en la parte en que choca con el sustrato se encuentra una película compacta de textura suave, color verde limón, creciendo sobre la roca. Las especies presentes son: 1 Chrysosaccus epilithicus; 2 Achnanthes lanceolata; 3 Schizothrix friesii; 4 Synedra ulna; 5 Mougeotia drouetii; 6 Oedogonium sp. 1; 7 Surirella ovalis.

PAP 326-1 (FCME) Forma de crecimiento: película filamentosa.

Al pie del paredón hay un goteo continuo que humedece una película filamentosa de color verde pasto y textura muy suave, que crece adherida a la roca. La película filamentosa es un crecimiento que se distingue porque a simple vista, o con ayuda de una lupa, puede verse la trama formada por los filamentos bien compactados. En este caso solamente hay tres especies: 1 Rhizoclonium hieroglyphicum; 2 Oedogonium sp. 1; 3 Mougeotia drouetii.

PAP 326-2 (FCME) Forma de crecimiento: tapete.

Al pie del paredón hay un goteo que humedece un tapete algal de color pardo oscuro con textura de fieltro; crece adherido a la roca. En el tapete, aunque puede estar compuesto de filamentos, no se distingue la trama (excepto cuando se observa al microscopio), a causa de la compactación de los filamentos. En paredones se manifiesta en lugares con poca humedad y a ello puede deberse la textura de fieltro. Puede tener una forma de vida epipélica o epilítica. Esta forma de crecimiento está integrada por las siguientes especies: 1 Oscillatoria lemmermannii; 2 Oscillatoria angustissima; 3 Rhopalodia gibba; 4 Microcystis pulverea; 5 Rhizoclonium hieroglyphicum; 6 Mougeotia 
drouetii; 7 Lyngbya allorgei; 8 Navicula lanceolata; 9 Navicula salinanum var. internedia; 10 Amphora ovalis; 11 Achnanthes lanceolata; 12 Gloeocapsa polydermatica; 13 Cosmarium margaritatum; 14 Oscullatoria geminata; 15 Synedra ulna; 16 Oocystis crassa; 17 Cocconeis placentula var. euglypta; 18 Surirella ovalis; 19 Epithemia argus; 20 Melosira sp.; 21 Lyngbya aerugineo-coerulea; 22 Lyngbya martensiana; 23 Chlamydocapsa ampla

PAP 328-1 (FCME) Forma de crecimiento: crecimiento hemisférico compacto.

En los puntos en que hay filtración de agua hay unos crecimientos hemisféricos compactos de color verde claro y textura suave; miden entre 1 y $2 \mathrm{~mm}$ de diámetro. Crecen sobre un sustrato lodoso muy compacto y está constituido por las siguientes especies: 1 Gloeothece rupestris; 2 Chroococcus turgidus; 3 Calothrix geitonos; 4 Oocystis crassa; 5 Cosmarium margaritatum; 6 Pediastrum integrum; 7 Mougeotia drouetii; 8 Navicula cincta; 9 Achnanthes exigua; 10 Nitzschia amphibia.

PAP 328-2 (FCME) Forma de crecimiento: flóculo mucilaginoso.

En los puntos en que hay filtración de agua, crece sobre el lodo un flóculo mucilaginoso de color verde claro y textura suave. Al microscopio se observó que en él hay dos asociaciones bien delimitadas que no pudieron distinguirse en el campo. La primera, que llamamos 328-2a está formada por: 1 Anabaena sp.; 2 Chroococcus varius; 3 Lyngbya muscicola; 4 Chroococcus multicoloratus; 5 Aphanothece caldarionum; 6 Denticula elegans; 7 Oocystis crassa; 8 Cosmarium margaritatum; 9 Cosmarium formosulum; 10 Pediastın integrum; 11 Cosmarium pseudopyramidatum; 12 Nitzschia amphibia; 13 Cladophora sp. La segunda, que denominamos 328-2b, está integrada por: 1 Microcystis marginata; 2 Gloeocapsa polydernatica; 3 Lyngbya muscicola.

HUAUTLA. Esta localidad se encuentra a $8 \mathrm{~km}$ del poblado del mismo nombre, en dirección SE ( $18^{\circ} 05^{\prime} 9.36^{\prime \prime}$ lat N; $96^{\circ} 47^{\prime} 30^{\prime \prime}$ lon O). A la orilla de la carretera, en el fondo de un pliegue del terreno se forma un paredón con sustrato rocoso sedimentario metamorfizado, (rocas blanquecinas de calizas dolomíticas, calcarenitas, lutitas y areniscas). El paredón mide aproximadamente $6 \mathrm{~m}$ de altura, el agua se reduce a un goteo continuo. Sobre la pared crece una escasa vegetación formada por helechos, principalmente una especie de Adiantum, varias especies de musgos y hepáticas. El panorama ficológico es muy pobre, formado por costras y escasos flóculos filamentosos. El pH es de 6 y la temperatura del agua es de $15^{\circ} \mathrm{C}$; su orientación es de $260^{\circ} \mathrm{SO}$ y está a 2000 msnm.

PAP 344 (FCME) Forma de crecimiento: costra.

Sobre la roca hay un crecimiento costroso humedecido continuamente por el goteo. La costra es de color verde oscuro y textura suave. En este paredón, distinguimos este crecimiento porque tiene un aspecto seco a pesar de que recibe humedad continuamente y porque no se desprende sin ayuda de algún instrumento como navaja o espátula, o con la uña. Se fragmenta al colectarla. Las especies que la integran son: 1 Tolypothrix tenuis 2 Lyngbya scottii; 3 Microchaete uberrima; 4 Spirogyra sp.; 5 Oedogonium sp. 1.

PAP 345 (FCME) Forma de crecimiento: flóculo filamentoso. 
En algunas partes de la pared humedecidas por el goteo, hay lodo depositado sobre el cual crecen unos flóculos filamentosos de color verde claro y textura suave. Son muy escasos. Están compuestos por las siguientes especies: 1 Oedogonium sp. 1; 2 Cymbella tumida; 3 Epithemia argus; 4 Navicula lanceolata; 5 Synedra uln a; 6 Epithemia adnata; 7 E. adnata var. minor; 8 Rhopalodia gibba; 9 Oscillatoria limosa.

KILOMETRO 24 Esta localidad está a $12 \mathrm{~km}$ de Huautla, en dirección SE, ( $18^{\circ} \mathrm{O} 2^{\prime} 47.24^{\prime \prime}$ lat $\mathrm{N} ; 9^{\circ} 45^{\prime} 57.36^{\prime \prime}$ lon O). Se trata de una pared de sustrato rocoso con pequeñas piedras sueltas; la roca es sedimentaria metamorfizada, del tipo descrito para la localidad de Huautla. Presenta escurrimiento de capa delgada de agua. La vegetación en este paredón es de musgos y hierbas pequeñas. El aspecto algal está constituido por películas y flóculos filamentosos. En los puntos en que se colectó hay un $\mathrm{pH}$ de 6.5 y la temperatura del agua es de $19^{\circ} \mathrm{C}$. Su orientación es $27.4^{\circ} \mathrm{NO}$ y su altitud es de 1725 msnm.

PAP 346-1 (FCME) Forma de crecimiento: flóculo filamentoso.

Sobre la pared crecen algunos manchones de musgos en el curso de la corriente. Entre estos y adheridos a ellos, es decir, epilíticos y epífitos, crecen unos flóculos filamentosos de color verde claro y textura suave. Las especies que determinamos en esta forma de crecimiento son: 1 Mougeotia drouetii; 2 Oedogonium sp. 3; Netrium digitus; 4 Oocystis crassa; 5 Cymbella minuta; 6 Cosmarium messochondrion; 7 Actinotaenium sp; 8 Bulbochaete sp.

PAP 347 (FCME) Forma de crecimiento: película filamentosa.

En el curso de la corriente crece una película filamentosa de color verde-azul y textura suave, epilítica, formada por las siguientes especies: 1 Mougeotia drouetii; 2 Navicula cincta; 3 Netrium digitus; 4 Navicula lanceolata; 5 Oedogonium sp. 2; 6 Synedra ulna; 7 Cymbella minuta; 8 Oscillatoria tenuis; 9 Cosmarium pachydermum var. pusillum; 10 Cosmarium pseudopyramidatum; 11 Achnanthes grimmei; 12 Mastogloia elliptica var. dansei; 13 Oscillatoria raoi; 14 Oocystis crassa; 15 Navicula pupula var. rectangularis.

PAP 348 (FCME) Forma de crecimiento: flóculo filamentoso.

Entre las rocas de la pared, en el curso de la corriente y en los puntos en que se deposita lodo, crecen flóculos filamentosos de color verde claro y de textura suave.

Están formados por: 1 Zygnema sp.; 2 Mougeotia drouetii; 3 Cymbella minuta; 4 Navicula lanceolata; 5 Mastogloia elliptica var. dansei; 6 Navicula salinarum var. intermedia; 7 Oedogonium sp. 1; 8 Gomphonema parvulum; 9 Achnanthes grimmei; 10 Cosmarium pseudopyramidatum; 11 Navicula exigua var. capitata; 12 Oscillatoria tenuis; 13 Merismopedia glauca.

SAN JUAN COATZOSPAN. Esta localidad se encuentra a 14 kilómetros de Huautla, en dirección SE (18 $3^{\prime} 04.12^{\prime \prime}$ lat N; $96^{\circ} 45^{\prime} 43.48^{\prime \prime}$ lon O). Se trata de una pared de roca sedimentaria también compuesta de calizas dolomíticas y calcarenitas, pero en esta zona hay además calizas puras y yesíferas. En este paredón no hay escurrimiento, sino que está humedecido por el agua que se filtra a través del sustrato. En la pared abundan musgos, hepáticas y líquenes. El panorama algal es exuberante, formado por flóculos 
mucilaginosos y filamentosos. $\mathrm{El} \mathrm{pH}$ es de 6 y la temperatura del agua es de $18^{\circ} \mathrm{C}$. Tiene una orientación de $18^{\circ} \mathrm{NE}$ y está a $1727 \mathrm{msnm}$

PAP 349-1 (FCME) Forma de crecimiento: flóculo mucilaginoso.

Humedecidos por el agua que se filtra, crecen unos flóculos mucilaginosos de color verde claro y textura suave. Crecen sobre el sustrato rocoso de manera abundante (una gruesa cubierta de 3-6 cm de espesor). Las especies presentes en este crecimiento son: 1 Aphanocapsa muscicola; 2 Chroococcus minor; 3 Gloeocapsa gelatinosa; 4 Navicula lanceolata; 5 Navicula salinarum var. intermedia; 6 Gloeocapsa decorticans; 7 Lyngbya limnetica; 8 Oocystis crassa; 9 Cosmarium pachydermum var. pusillum; 10 Chroococcus turgidus; 11 Gomphonema parvulum; 12 Cymbella minuta; 13 Achnanthes Grimmei; 14 Lyngbya aerugineo-coerulea 15 Cosmarium messochondrion; 16 Rhopalodia gibba; 17 Cosmarium pseudopyramidatum; 18 Denticula elegans.

PAP 349-2 (FCME) Forma de crecimiento: flóculo filamentoso.

Junto a los flóculos de la muestra anterior, también humedecidos por el agua que se filtra, crecen unos flóculos filamentosos de color verde claro y textura suave. Se encuentran sobre el sustrato rocoso y están constituidos por: 1 Mougeotia drouetii; 2 Oocystis crassa; 3 Navicula lanceolata; 4 Navicula salinarum var. intermedia; 5 Chroococcus turgidus; 6 Lyngbya rivularianum; 7 Cymbella minuta; 8 Synedra ulna; 9 Cosmarium pseudopyramidatum; 10 Rhopalodia gibba; 11 Nitzschia amphibia; 12 Oedogonium sp. 1.

\section{DISCUSIÓN}

Las formas de crecimiento de las algas (crecimientos visibles), podrían compararse con las formaciones de plantas vasculares porque son la apreciación de una comunidad a primera vista, pero las formas de crecimiento en las algas son la convergencia de varios niveles de organización con una forma de vida. Para explicarlo tomemos como ejemplo la muestra PAP 328-2a de los datos que se presentan en este trabajo. Esta forma de crecimiento es la reunión de varias especies con un nivel de organización cenobial y varias especies unicelulares, que crecen de manera epilítica (forma de vida), inmersas en el escurrimiento. En este caso la manifestación visible de las especies tiene una forma de crecimiento de flóculo mucilaginoso y las especies tienen una forma de vida epilítica. En otro ambiente, las mismas especies podrían ser planctónicas, e integrar natas como forma de crecimiento. Las formas de crecimiento son importantes porque a los ojos de cualquier observador, cierto tipo de natas identifican a los charcos y cierto tipo de flóculos identifican a los paredones. En otras palabras, las formas de crecimiento son importantes para tipificar un ambiente porque para cada ambiente existen ciertas formas de crecimiento que tienen una estrecha relación con su fisiografía.

Por ello podemos utilizar estas formas para reconocer diferencias de ambientes similares en distintos tiempos o espacios, y también para reconocer semejanzas entre ambientes distintos. Esta es una de las razones por las que las descripciones de campo de distintos autores, para lugares y épocas diferentes son tan parecidas, tanto entre si 
como con las nuestras (Hustedt, 1953; Johansen et al. 1980; Rushforth et al., 1976; Rushforth et al., 1980). En la tipificación de un ambiente algal, que es parte de su caracterización florística, además de recurrir a las formas de crecimiento, tenemos que considerar el papel de las especies. Como puede verse en los resultados de este trabajo, la sola relación presencia-ausencia de la flora no permite integrarla con las características fisiográficas porque las formas de crecimiento son grupos funcionales, es decir, son la conjunción de manifestaciones diferenciales de distintos patrones estructurales básicos en coincidencia con factores mesológicos particulares para el momento de coincidencia; con esto queremos decir que si bien las formas de crecimiento son típicas de manera independiente del momento, (por ejemplo, del momento en que se realiza la colecta sin importar el número de momentos), las especies no lo son, porque la flora puede cambiar no solo en composición y abundancia sino en los conjuntos de características de esas especies en cada momento de manifestación (manifestación diferencial). Puede cambiar por ejemplo la fase del ciclo de vida de cualquiera de las especies presentes (y con ello posiblemente el nivel de organización); puede cambiar el vigor de los individuos, su abundancia, su estado fenológico; el número, tipo y etapa de las estructuras reproductoras y un enorme número de características que sería imposible mencionar. La flora de cualquier localidad, si la basamos sólo en la presencia eventual de las algas, dice mucho en términos de inventario, pero poco en términos ecológicos y justamente el análisis de grupos funcionales debe ser con base en criterios ecológicos. Los valores de abundancia relativa que asignamos a las especies que constituyen cada forma de crecimiento tienen la finalidad, en este trabajo, de resaltar que la importancia de las especies como integrantes de una forma de crecimiento, no está dada sólo por su presencia sino por ciertas cualidades de manifestación, como la abundancia por ejemplo. Manejamos sólo la abundancia por dos razones, la primera es lo evidente que resulta que una forma de crecimiento está determinada por los niveles de organización de las especies que la integran y por la abundancia relativa de las mismas. Por ejemplo, un flóculo filamentoso esta determinado por cualquier especie con un nivel de organización filamentoso por lo menos en alguna etapa de su ciclo de vida (cuadro 2). La segunda razón es que no es fácil probar, por intuitivamente claro que parezca, que realmente están en juego todas las características o cualidades de manifestación que hemos mencionado, si no podemos precisar cuál, cuándo y en qué grado influyen. Sería necesario establecer una unidad de medida para evaluar estas cualidades y facilitar un análisis que permita uniformar criterios para evaluar comparativamente el impacto de cada especie en las distintas formas de crecimiento, pero parece clara la importancia de utilizar las formas de crecimiento para tipificar un ambiente. Como se observa en los resultados, los crecimientos más comunes en los paredones son de tipo floculoso. Así mismo hemos mencionado que las descripciones de la cobertura algal y la fisiografía de paredones en diferentes latitudes son muy similares, de manera que muy posiblemente las formas de crecimiento están estrechamente vinculadas con las características fisiográficas del ambiente. Por tanto, definimos a los paredones como un ambiente algal, 
conno escurrimientos verticales con muy poca cantidad de agua, en los que los flóculos filamentosos y mucilaginosos son las formas de crecimiento típicas (cuadro 1). Aunque estos crecimientos no son exclusivos de los paredones, parece haber una constancia de su manifestación y depende de las condiciones fisiográficas de cada paredón, por ejemplo grado de humectación, las características de cómo y cuánto están presentes estos flóculos, y la posibilidad de mayor o menor diversidad de otros tipos de crecimientos (como tapetes, películas, costras, etcétera.).

AGRADECIMIENTOS. Los autores queremos agaradecer al Dr. Rodolfo Dirzo Minjarez del Centro de Ecología, UNAM, la revisión del manuscrito y sus valiosos comentarios.

\section{LITERATURA CITADA}

DÍAZ, B. M., A. DONALDSON, M. K. HARGREAVES, N. T. H. HOLMES, M. K. HUGHES, P. J. SAY y B. A. WHITTON. 1976. A computer orientated recording system for plants in flowing water. University of Durham. 55p.

DONALDSON, A. 1978. Studies on the terrestrial and freshwater algae of the Aldabra atoll. $\mathrm{Ph} . \mathrm{D}$. Thesis. University of Durham, Durham, England. 478p.

DONALDSON, A. B. A. WHITTON. 1977. Algal flora of freshwater habitats on Aldabra. Atoll Research Bull. 215:1-26.

HUSTEDT, F. 1953. La flora de diatomeas en paredones sobrehumedecidos en El Salvador. Comunicaciones 2(5-6):129-138.

JOHANSEN, J. R., S. R. RUSHFORTH y J. D. BROTHERSON. 1980. The algal flora of the Navajo National Monument. Arizona. J. Phycol. 16 (Suppl.):22.

MIRANDA, F. 1948. Datos sobre la vegetación de la cuenca alta del Papaloapan. An. Inst. Biol. Mex. 19:333-364.

MOSIÑO, P. A. 1977. Metereología y climatología. En: Tamayo, J. L. y Beltrán, E. (Eds.) Recursos naturales de la cuenca del Papaloapan, SARH-Comisión del Papaloapan, IMERNAR, México, D.F. Tomo I. pp.63-92.

RUSHFORTH, S. R., S. D. BURTON, J. R. JOHANSEN y J. A. GRIMES 1980. The bacterium Thiploca ingarica on wet walls in Zion National Park, Utah. Great Basin Naturalist. 40 (1):98-100.

RUSHFORTH, S. R., L. L. CLAIR, T. A. LESLIE, K. H. THOME y D. C. ANDERSON 1976. The algal flora of two hanging gardens in South-eastern Utah. Nova Hedwigia. 27:231-323.

SALAS, G. P. 1977. Recursos no renovables. En: Tamayo, J. L. y Beltrán, E. (Edrs.) Recursos naturales de la cuenca del Papaloapan, SARH-Comisión del Papaloapan, IMERNAR, México, D.F. Tomo I. pp.221-250.

WHITTON, B. A., B. M. DIAZ y N. T. H. HOLMES. 1979. A computer orientated numerical coding system for algae. Br. Phycol. J. 14:353-360.

STEEN, E.B. 1971. Dictionary of biology. Barnes and Noble. 630p. 